\title{
MICROSCOPIC EXAMINATION OF COTTON FIBERS IN CUPRAMMONIUM HYDROXIDE SOLUTIONS *
}

\author{
By Charles W. Hock and Milton Harris ${ }^{1}$
}

ABSTRACT

During treatment of cotton with cuprammonium hydroxide solutions, the cellulose dissolves, leaving residues which vary in amount and in structure, depending upon the degree of purification of the fibers. The undissolved residue from raw and from dewaxed fibers consists principally of fragmented shells that formed the outer surface of the fibers, and, to a lesser extent, of material from the lumen. Both of these residues are isotropic and stain deeply with ruthenium red. Fibers from which both wax and pectic substance have been removed dissolve in cuprammonium reagent, leaving only a very small amount of isotropic residue which exhibits no definite cytological structure.

When a steady flow of cuprammonium hydroxide solution is applied to fibers mounted on a slide, the initial swelling of the fibers is followed by the appearance of small ellipsoidal particles which range from 1 to $2 \mu$ in size, appear bright between crossed nicols, and become dark in color when stained with iodine and sulfuric acid. Identical particles appear, however, when cuprammonium hydroxide solution is drawn under the cover glass, in the absence of fibers. These particles are formed both in the presence and absence of cotton, and result from the exposure of the cuprammonium hydroxide reagent to air. They are washed under the cover glass by the flow of cuprammonium hydroxide and become associated with any fiber residue on the slide. Formation of these particles may be prevented by using a specially constructed cell which prevents exposure of the reagent. Under these conditions, no particles were observed during the slow passage, even for prolonged periods, of the cuprammonium reagent over the cotton fibers.

Two cuprammonium hydroxide solutions, one prepared according to ACS specification, and the other higher in ammonia content, were used in this investigation. It was found that particles formed much more quickly in the former, and accordingly make it less desirable for microscopic studies of the behavior of cotton fibers in cuprammonium reagent.

\section{CONTENTS}

I. Introduction

II. Materials _._.

III. Experimental procedure and results

IV. References._. 748

\section{INTRODUCTION}

The viscosity of cuprammonium hydroxide solutions of cotton has frequently been related to the quality of the fiber, especially as regards tensile strength. It has been shown, for example, that chemical treatments which modify the properties of the fibers in such a way as

* This paper was presented before the Cellulose Division of the 99th meeting of the American Chemical Society, Cincinnati, Ohio.

1 Research Associates at the National Bureau of Standards, representing the Textile Foundation. 
to impair their tensile strength also bring about a lowering of the viscosity in cuprammonium hydroxide solutions $[1,2] .^{2}$ On the basis of the molecular chain theory for the structure of cellulose, this decrease in tensile strength and the accompanying increase in cuprammonium hydroxide fluidity may be regarded as having resulted from a shortening in chain-length of the cellulose molecule. Recently, Farr and her collaborators [3, 4, 5] have brought forth a new explanation based principally on microscopic observations. According to these investigators, the cellulose component of cotton fibers does not dissolve in cuprammonium solution but maintains a visible state of aggregation in the form of diminutive cellulose particles, each of which is surrounded by cementing material which swells in cuprammonium solutions, thereby giving rise to a gel-like structure upon which many of the characteristic properties of the dispersion depend. On the basis of this interpretation, it follows that the reduction of viscosity through processing or deterioration of the fibers may be regarded as the result of a direct effect upon the viscosity-producing power of the cementing material.

Since measurements of the viscosity of cuprammonium hydroxide solutions of cotton are widely used to evaluate some of the characteristics of the fiber, and since the above concepts lead to widely different interpretations of the behavior of cotton in this solution, it appeared advisable to undertake a new investigation of the microscopically observable changes which cotton fibers undergo in this reagent.

\section{MATERIALS}

The cotton used in these experiments was Gossypium hirsutum L, Missdel-7 variety, grown at the Delta Experiment Station at Stoneville, Miss., and furnished by the Bureau of Agricultural Economics, United States Department of Agriculture. It had been ginned and twice carded, but had received no chemical treatments. In the present paper these fibers are designated as raw fibers. The natural waxes were removed from the raw fibers by extraction with alcohol and ether for 24 hours each. These fibers are termed dewaxed fibers. A portion of the dewaxed material was further purified by extraction for 6 hours with a boiling 1-percent solution of sodium hydroxide, according to the method recommended for the preparation of standard cellulose. The procedure was essentially the same as that described by Corey and Gray [6], except that the apparatus of Worner and Mease [7] was employed. Fibers treated in this way were shown by the method of Whistler, Martin, and Harris [8] to be free of pectic substance. These fibers, from which both wax and pectic substance were removed, are designated depectinized fibers.

5. Two solutions of cuprammonium hydroxide were used in the experiments. One, made according to the recommendations of Mease [9], is essentially the same as that set forth by the Fabrics Research Committee (London) [10], except that the higher concentration of ammonia originally recommended by Clibbens and Geake [11] was used. This solution contained $240 \pm 5 \mathrm{~g}$ of ammonia $\left(\mathrm{NH}_{3}\right), 15 \pm 0.1 \mathrm{~g}$ of copper, and $1.0 \mathrm{~g}$ of sucrose, per liter. The concentration of nitrite was less than 0.5 percent. In the present paper this solution will hereafter be

\footnotetext{
-1 igures in brackets indicate the literature references at the end of this paper.
} 
referred to as cuprammonium hydroxide solution A. A second cuprammonium hydroxide solution, prepared according to the specification of the Division of Cellulose Chemistry of the American Chemical Society [12] was also used. It contained $30 \pm 2 \mathrm{~g}$ of copper, $165 \pm 2 \mathrm{~g}$ of ammonia, and $10 \mathrm{~g}$ of sucrose, per liter. In the present paper this solution will bereafter be referred to as cuprammonium hydroxide solution $B$. When not in actual use, all solutions of cuprammonium hydroxide were kept under nitrogen in tightly stoppered bottles in the refrigerator. Unless stated otherwise, both solutions $A$ and $B$ were used in each of the experiments in this investigation.

\section{EXPERIMENTAL PROCEDURE AND RESULTS}

Raw, dewaxed, and depectinized fibers were placed, without any mounting medium, on microscope slides, and then examined microscopically as cuprammonium hydroxide solution was drawn under the cover glass. In other cases the fibers were mounted in distilled water, or in ammonium hydroxide, and the cuprammonium sulution was added as above. The water and ammonium hydroxide appeared to act merely as diluents, thereby retarding the solvent action of the cuprammonium hydroxide solution. The behavior of the fibers in the cuprammonium reagent was observed microscopically, using ordinary and dark-field illumination and crossed nicols.

Raw cotton fibers, upon addition of the reagent, immediately began to swell and twist, often forming balloon-like structures (fig. 1, $A$ and $B)$. After a few minutes, the inner portion of the fiber appeared to be completely dissolved, leaving transparent shells which originally formed the outer surface of the fibers (fig. 1,C). Likewise, a small amount of material from the lumen did not dissolve (fig. 1, $B$ ). This behavior was especially striking when observed with crossed nicols. The fibers, originally bright against a black background, became dimmer as the cellulose was dissolved by the reagent. After a few minutes, the field was black, which indicated that all birefringent material had disappeared. When the same preparation was then examined in ordinary light, it was found to contain many of the shells described above.

Dewaxed cotton fibers presented essentially the same picture as raw fibers. Dissolution of the inner part of the fiber took place in a few minutes, leaving residues (fig. 2) similar to those of the raw fibers (fig. 1, C), but less dense and bulky, and often more torn and fragmented than the latter. With crossed nicols the results were the same as with raw cotton; that is, the strongly birefringent cellulose was dissolved rapidly, leaving an insoluble, isotropic material.

Depectinized cotton fibers dissolved in cuprammonium $\$$ hydroxide solution, leaving only a very small amount of isotropic residue which consisted of small irregular bits of material (fig. 3). This residue exhibited no definite cytological structure, such as that shown by the shells of raw and of dewaxed fibers. (Compare with fig. 1, $C$ and fig. 2.)

The isotropic shells of raw and of dewaxed fibers and the isotropic material from the lumen did not stain blue when given the iodinesulfuric acid test for cellulose, but they stained deeply with ruthenium red [13]. Although ruthenium red is not a specific stain for pectic 
substance, native pectic compounds invariably take a red color in the presence of this dye [14].

In the above experiments the effects of cuprammonium reagent on the different samples of cotton could be observed for only relatively short periods. In order to make observations over longer periods, samples of raw, dewaxed, and depectinized cotton fibers were placed in separate vials of cuprammonium hydroxide solution. At intervals thereafter, samples from each of the vials were removed and viewed microscopically, using ordinary and dark-field illumination and crossed nicols. During the first day, the preparations were examined every few hours; during the remainder of the first week, examinations were made once each day; and thereafter, once each week for a period of 6 weeks. After the initial dissolution of cellulose, which appeared to be complete in a few minutes, there was no further change in microscopic appearance of the undissolved material. The solutions of raw and of dewaxed cotton showed nothing but isotropic residues, whereas the samples containing depectinized cotton were practically free of residue.

These experiments indicate that pectic substance is a constituent of the insoluble shells which remain when cotton fibers are treated with cuprammonium hydroxide solutions. This conclusion is substantiated by direct chemical evidence presented elsewhere [2]. It also appeared from these experiments that the cellulose portion of the fibers dissolved in cuprammonium reagent, leaving no microscopically resolvable particles of the type described by Farr [3], who has stated, however, that the particles are not visible as ordinarily observed, since they are obscured to the point of invisibility by a matrix of colloidal cementing material which surrounds them. It is stated further that by maintaining a steady flow of cuprammonium hydroxide over the fibers the matrix may be removed, thereby rendering the particles visible. It might have been expected, however, that the particles would have been visible in the depectinized fibers, since these fibers left an almost negligible amount of visible colloidal material after treatment with cuprammonium reagent.

Since particles were not observed, it appeared advisable to repeat Farr's experiments exactly as they are described. Raw cotton fibers were mounted in distilled water. The water was then gradually replaced with cuprammonium hydroxide solution by the following procedure: Small drops of the reagent were applied to one edge of the cover glass. The solution was then drawn under the cover glass by means of very narrow strips of filter paper placed at the opposite side. Swelling of the fibers ensued, resulting in the formation of some "balloons" (fig. 1, $A$ and $B$ ) which eventually disappeared, leaving residues like those described earlier. However, as the flow of fresh reagent was continued, small particles appeared (fig. 4, $A$ ).

The same technique was then applied to depectinized cotton fibers. As previously described, the cellulose appeared to dissolve upon addition of the cuprammonium hydroxide, leaving only a very small amount of insoluble residue. Nevertheless, as with raw fibers, particles became evident when a steady flow of the reagent was maintained.

It was noted, however, that identical particles appeared, even in the absence of fibers, when the steady flow of cuprammonium hydroxide was continued. The results were the same whether cuprammonium 

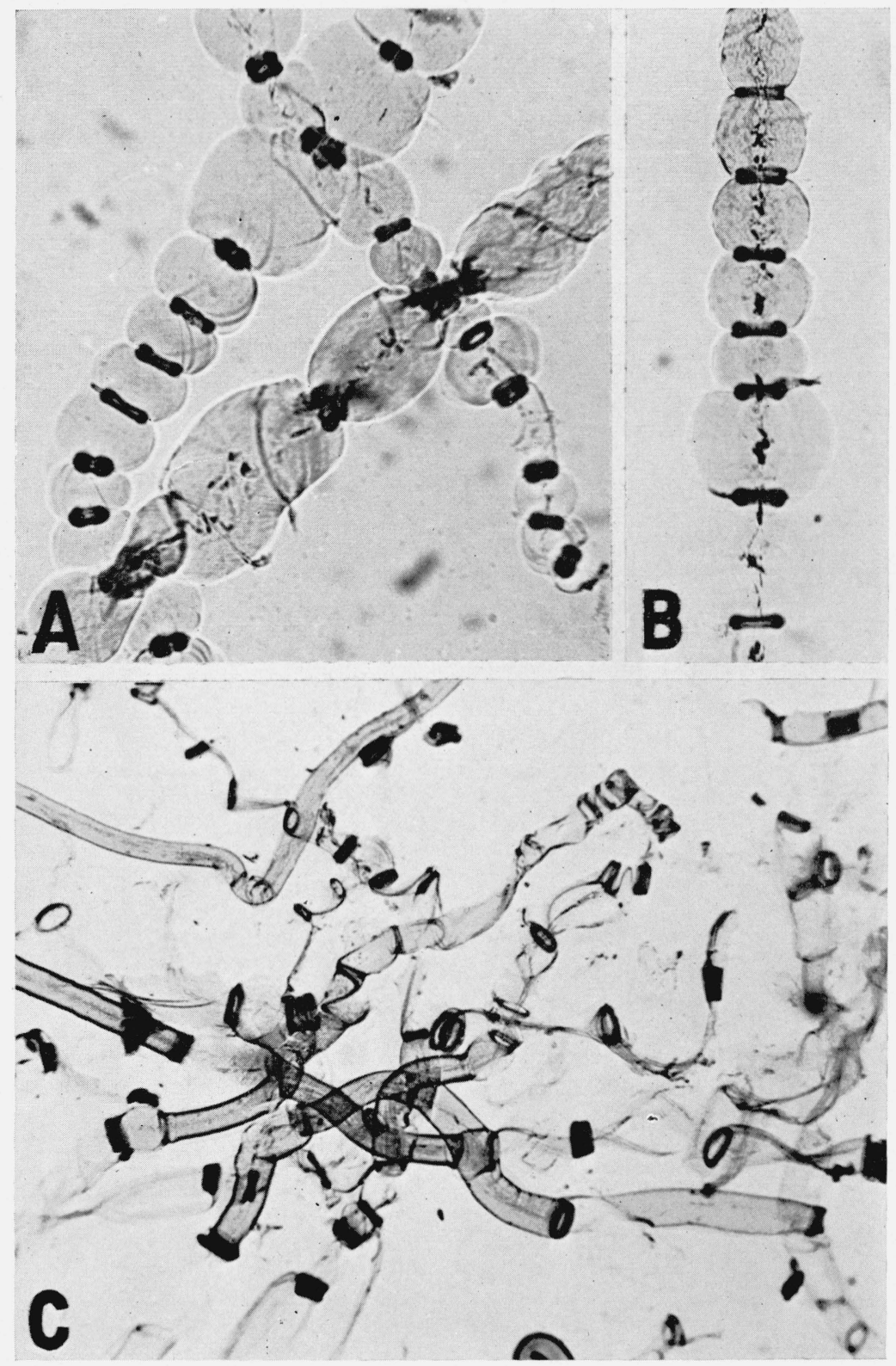

FigURE 1.-Raw cotton fibers in cuprammonium hydroxide solution.

$A$, fibers swollen to form "balloons"; $B$, typical string of "balloons," showing deeply stained material in the lumen and at the constrictions between the swellings; $C$, residue which remains after the cellulose of the fibers has dissolved in the reagent.

Fibers stained with ruthenium red. Magnification $\times 120$. 


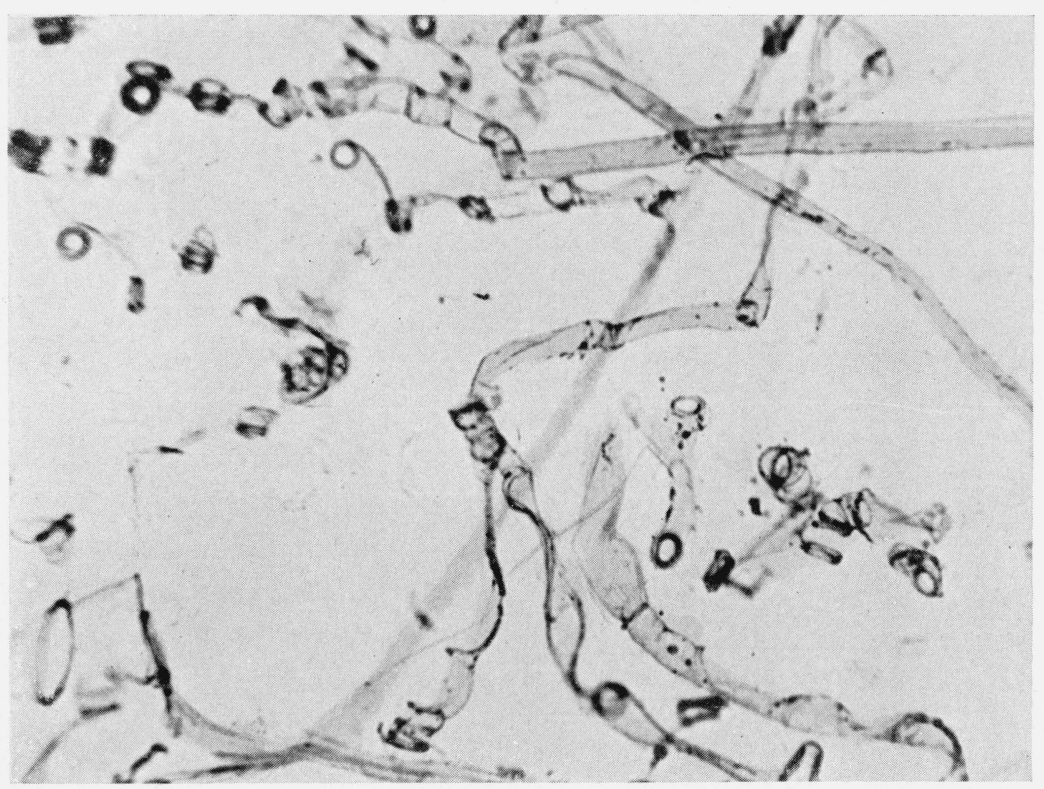

FIGURE 2.-Dewaxed cotton fibers in cuprammonium hydroxide solution.

Residue which remains after the cellulose of the fibers has dissolved in the reagent. Fibers stained with ruthenium red. Magnification $\times 120$. 


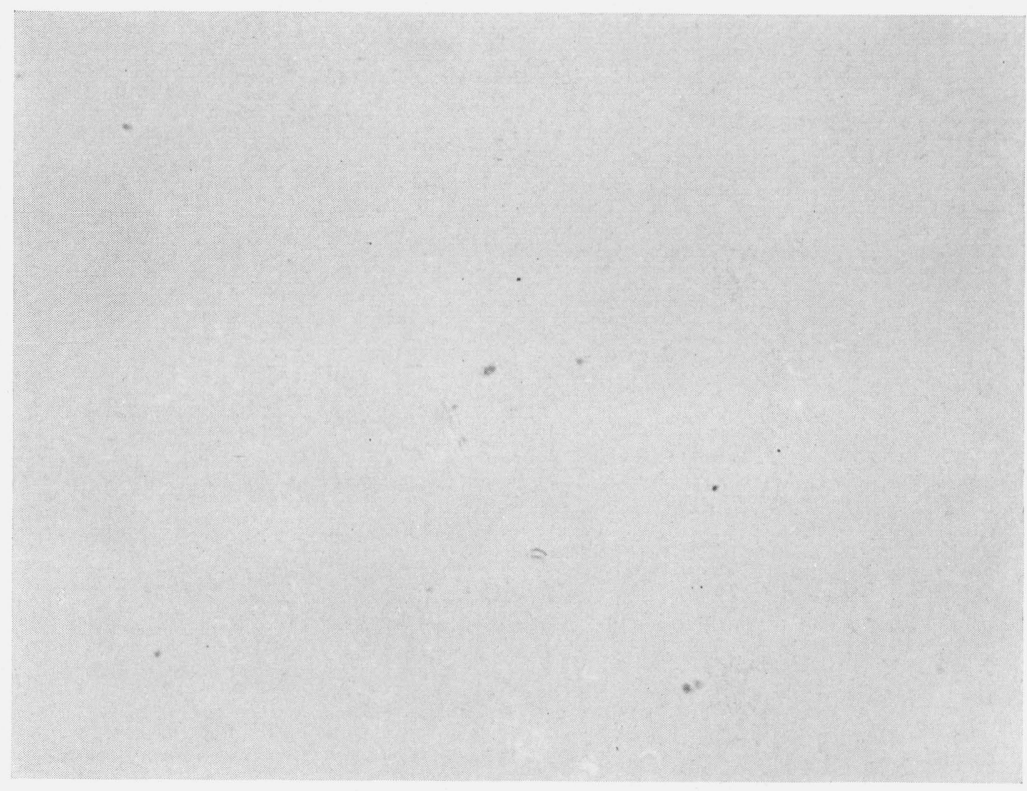

FiguRe 3.-Depectinized cotton fibers in cuprammonium hydroxide solution. Residue which remains after the cellulose of the fibers has dissolved in the reagent. Magnification $\times 120$. 

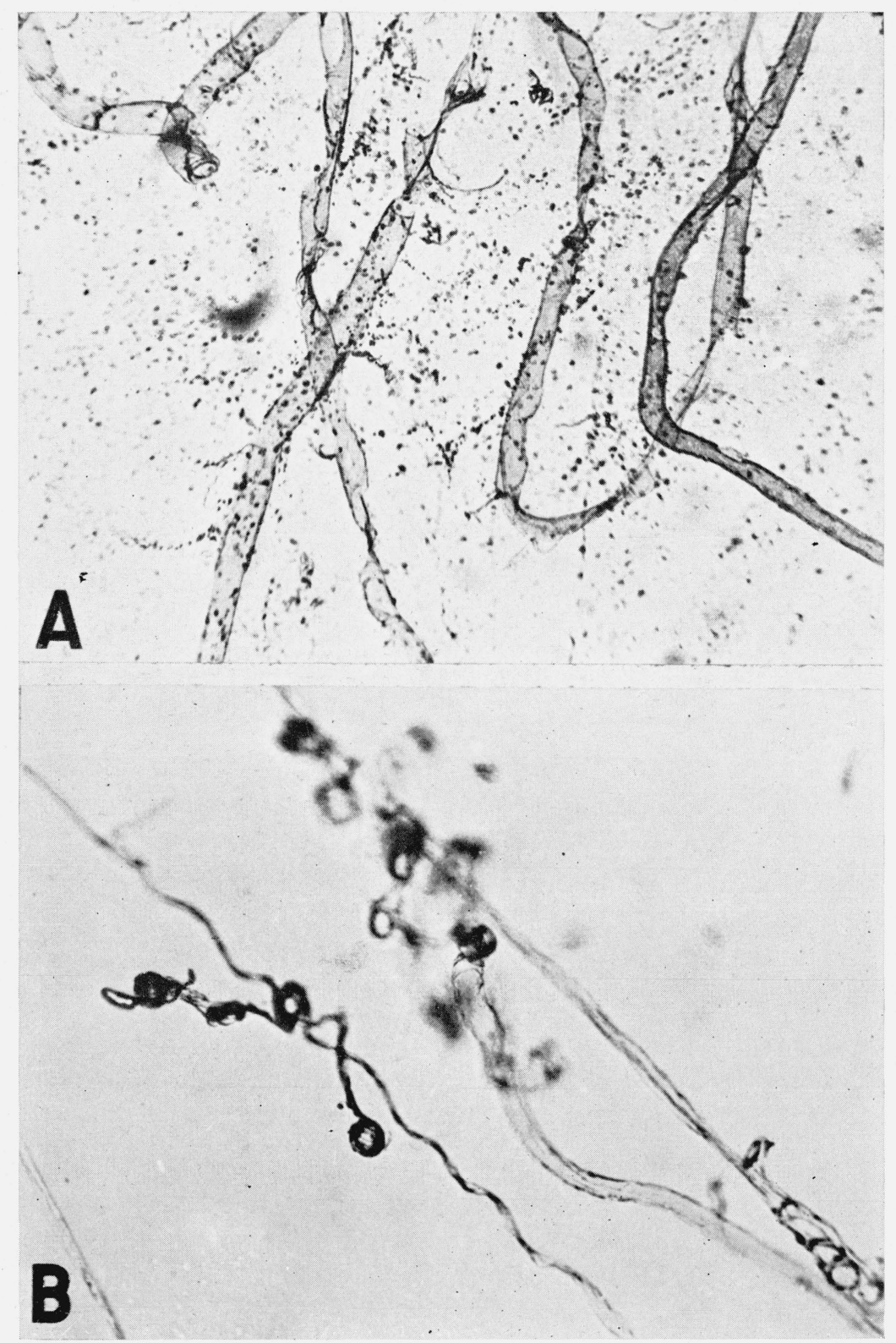

FIGURE 4.-Fibers treated with a steady flow of cuprammonium hydroxide solution. $A$, residue of raw fibers after a continuous flow of the reagent was maintained over them. Particles, formed from the cuprammonium solution, are seen among the residue. $B$, raw cotton fibers after treatment with cuprammonium solution for 1 hour, under conditions which prevented exposure of the reagent. Magnification $\times 120$ 


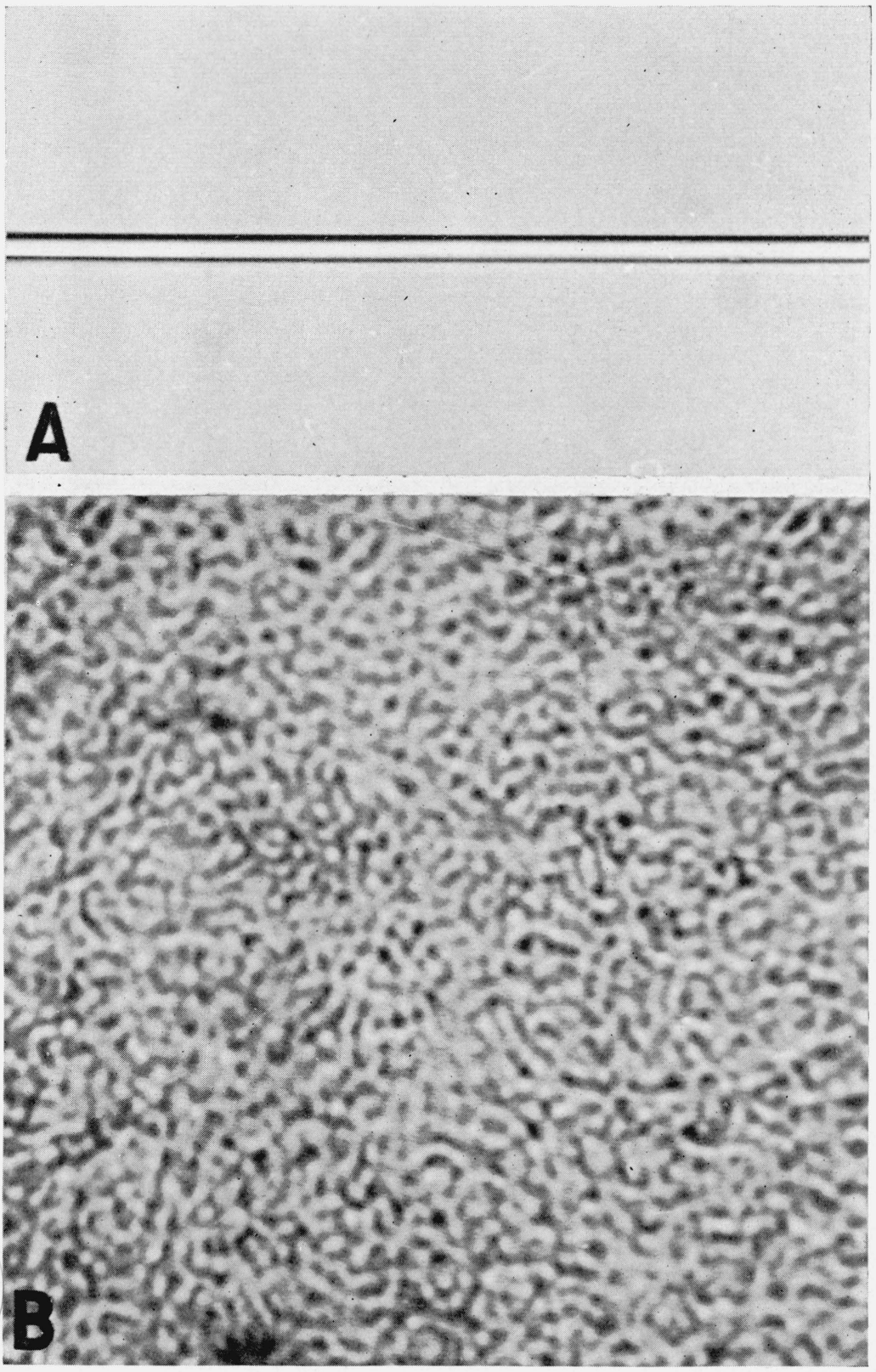

FIGURE 5.-Cuprammonium hydroxide solution: no cellulose present.

$A$, fine strand of glass rod in focus in the solution. The latter has not been exposed to allow formation of particles. Magnification $\times 500$. B, particles formed upon exposure of the reagent to air. Magnification $\times 1500$ 

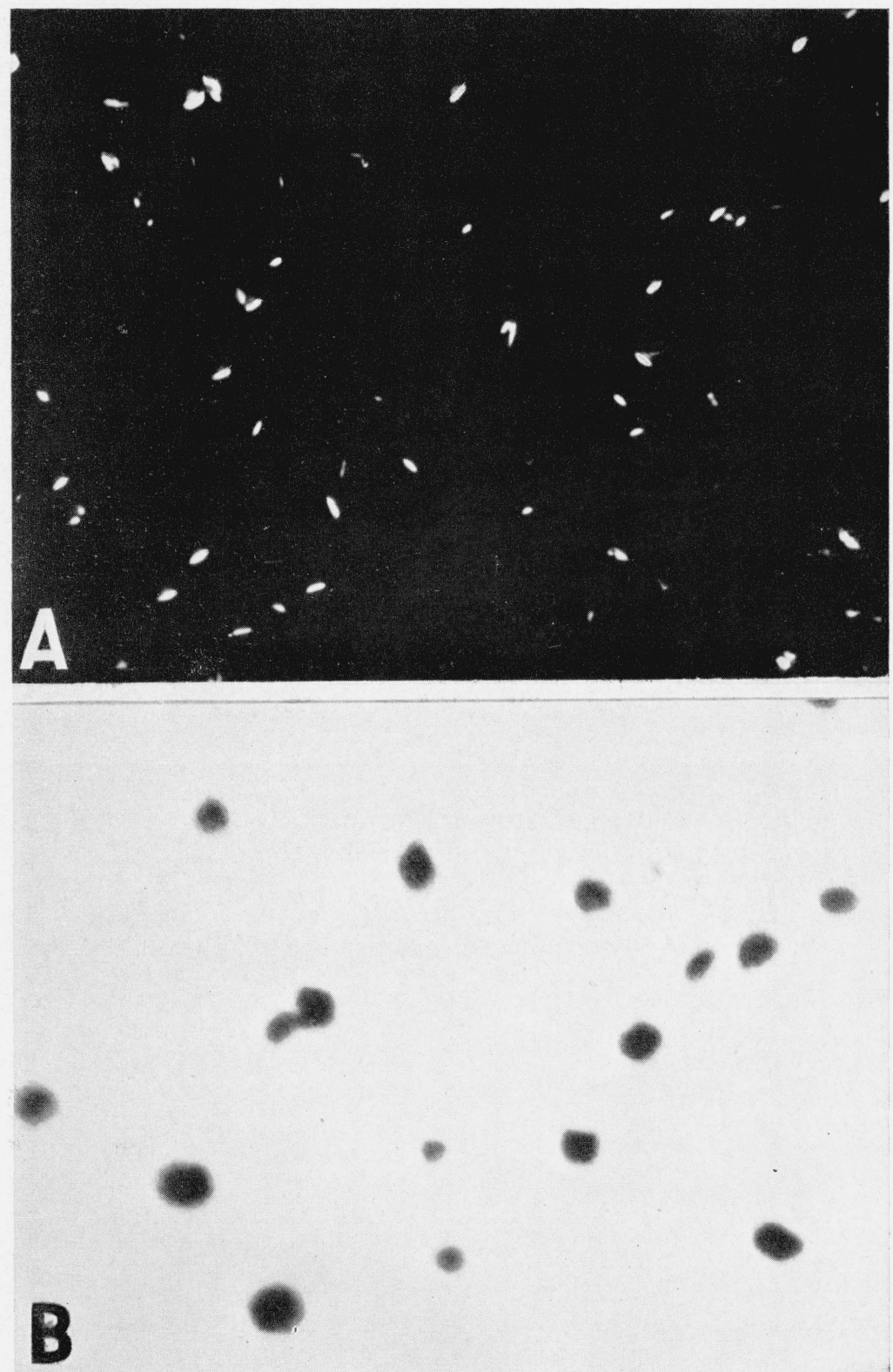

FIGURE 6.-Particles formed in cuprammonium hydroxide solution, in the absence of cellulose.

$A$, particles as viewed between crossed nicols. $B$, Particles which appear after the addition of iodine and sulfuric acid. Magnification $\times 1500$. 
solution $A$ or $B$ was used. In both cases, the particles appeared to be ellipsoidal and ranged from 1 to $2 \mu$ in size. They were birefringent and showed extinction when rotated between crossed nicols. When given the iodine-sulfuric acid test for cellulose, dark particles, somewhat larger than the original ones, were observed. The photomicrographs (figs. $5 B, 6 A, 6 B$ ) of these particles greatly resemble those shown by Farr [3]. All of these photomicrographs are of cuprammonium hydroxide solutions which contained no cotton fibers. In all cases the original solutions were clear (fig. $5, A$ ), and particles appeared only after the solution was exposed to the air, as in drawing it under the cover glass.

Further examination of the cuprammonium solutions showed that a precipitate was always formed when this reagent was exposed. Farr [3] has stated that a steady flow of the solution had to be maintained in order to prevent crystallization of the copper salt. In the present investigation, it was found that regardless of the care exercised in this experiment, particles were always obtained when the cuprammonium solution was allowed to flow under the cover glass according to the described technique.

The experiments in which a continuous flow of cuprammonium reagent was maintained over the fibers were then repeated under conditions which prevented exposure of the reagent to air. In order to do this a sealed mount, constructed as follows, was employed. A ring consisting of a mixture of paraffin and vaseline was made in the center of a microscope slide, and a few cotton fibers were then placed in the enclosure. A hypodermic syringe was arranged in such a way that the tip of the needle extended just inside the ring, on the opposite side of which a small opening was made. A cover glass was then placed over the fibers and the ring, and further sealed to the slide by applying a viscous solution of methyl methacrylate in acetone, to the edges of the cover glass. The microscope slide and syringe were fastened to a sheet of stiff cardboard so that the whole apparatus could be placed on the stage of the microscope.

The syringe was filled with cuprammonium hydroxide solution. By applying pressure to the plunger of the syringe, the solution was passed slowly over the fibers, which were mounted either dry or in distilled water. The liquid was allowed to flow through the cell and out the small opening in the ring. During this process the behavior of the fibers was continuously observed with the microscope.

Using this specially constructed cell, which prevented exposure of the reagent, fibers were treated with cuprammonium hydroxide solutions $A$ and $B$. It was found that a steady flow of solution $A$ could be passed over the fibers for more than an hour without the appearance of particles (fig. 4, B). As the reagent started to flow through the cell, the frequently observed reaction took place. The fibers began to swell, thereby giving rise to typical balloon-like structures, which eventually appeared to be optically clear. Moreover, as the flow of cuprammonium hydroxide was continued, no particles appeared, even after prolonged passage of the reagent.

When solution $B$ was used, some particles always appeared, even in control mounts which contained no fibers. In all cases in which the solutions were exposed to the air, particles formed more readily with solution $B$ than with $A$, probably because of the higher copper and lower ammonia concentrations of the former. Because of this 
fact, solution $A$ was found to be much more desirable for microscopic studies of the behavior of cotton fibers in cuprammonium reagent.

The results of this investigation indicate that microscopically resolvable particles of cellulose do not persist when cotton fibers are dispersed in cuprammonium hydroxide solutions. Particles are obtained, however, even in the absence of fibers, upon exposure of the reagent to air.

\section{REFERENCES}

[1] D. A. Clibbens and B. P. Ridge, J. Textile Inst. 19, T394 (1928).

[2] R. L. Whistler, A. R. Martin, and M. Harris, J. Research NBS 24, 555 (1940) RP 1299.

[3] W. K. Farr, Contrib. Boyce Thompson Institute 10, 71 (1938).

[4] W. A. Sisson, Contrib. Boyce Thompson Institute 10, 113 (1938).

[5] J. Compton, Contrib. Boyce Thompson Institute 10, 57 (1938).

[6] A. B. Corey and H. L. Gray, Ind. Eng. Chem. 16, 853, 1130 (1924).

[7] R. K. Worner and R. T. Mease, J. Research NBS 21, 609 (1938) RP1146.

[8] R. L. Whistler, A. R. Martin, and M. Harris, J. Research NBS 24, 13 (1940) RP1268.

[9] R. T. Mease, J. Research NBS 22, 271 (1939) RP1179.

[10] Report of the Fabrics Research Committee, Department of Scientific and Industrial Research, His Majesty's Stationery Office, London (1933).

[11] D. A. Clibbens and A. Geake, J. Textile Inst. 19, T77 (1928).

[12] American Chemical Society. Committee on the Viscosity of Cellulose of the Division of Cellulose Chemistry. Ind. Eng. Chem., Anal. Ed. 1, 49 (1929).

[13] L. Mangin, Compt. rend. 116, 653 (1893).

[14] D. B. Anderson and T. Kerr, Ind. Eng. Chem. 30, 48 (1938).

Washington, March 1, 1940. 